\title{
Realization of a compact optical interconnect on Silicon by heterogeneous integration of III-V
}

\author{
T. Spuesens, J. Bauwelinck, P. Regreny and D. Van Thourhout
}

\begin{abstract}
We present a compact optical interconnect on a silicon-on-insulator platform consisting of a directly modulated microdisk laser and detector connected via a silicon waveguide. A single III-V epitaxial structure that contains layers for both the laser and detector ensures that dense integration becomes possible. All fabrication steps for the laser and detector, except for the detector mesa, are carried out simultaneously. The microdisk laser has a threshold current of $0.45 \mathrm{~mA}$ and a slope efficiency of $57 \mu \mathrm{W} / \mathrm{mA}$. The responsivity of the detectors is 0.69 A/W. The full optical link has a static efficiency of $3 \%$ and a bandwidth of 7.6 GHz. A large signal modulation was applied and a $10 \mathrm{~Gb} / \mathrm{s}$ bit pattern could be resolved.
\end{abstract}

Index Terms - Heterogeneous integration, microdisk laser (MDL), optical interconnect, photonic integration, silicon photonics, waveguide photodetector.

\section{INTRODUCTION}

$\mathrm{O}$ $\mathrm{N}$-CHIP data rates are increasing rapidly and will continue to do so in the coming years. Not only speed limitations but also power dissipation is becoming a major point of concern in electrical interconnects. Optical interconnects have been proposed as a possible alternative to the electrical interconnects. To be a valid alternative for electrical interconnects, the optical link needs to have at least the same bandwidth, but also low power consumption and a compact size are required since thousands of these links must be integrated on a single chip [1].

Silicon photonics has emerged as a potential platform for optical interconnects because of its CMOS compatible fabrication process and the ability to make compact and complex circuits. Although silicon is not suited as a light emitter or detector in the infrared, several approaches have been demonstrated to integrate lasers and detectors on a silicon platform [2-5]. For efficient communication across the chip dense integration of optical transceivers, consisting of lasers or

Manuscript received March 7, 2013. This work was supported by the European FP7 ICT WADIMOS project. The work of T. Spuesens is supported by the Institute for the Promotion of Innovation through Science and Technology (IWT) under a specialization grant.

T. Spuesens, J. Bauwelinck and D. Van Thourhout are with the INTEC department, Ghent University-imec, 9000 Ghent, Belgium (e-mail: thijs.spuesens@ intec.ugent.be; dries.vanthourhout@intec.ugent.be).

P. Regreny is with the Institut des Nanotechnologies de Lyon (INL), INL Unité Mixte de Recherche (UMR) 5270, Centre National de la Recherche Scientifique (CNRS), Ecole Centrale de Lyon, Université de Lyon, F-69134 Lyon, France. modulators and detectors, is an important requirement. This can be achieved by using e.g. silicon modulators and germanium detectors [6]. However, these modulators have generally high insertion loss. When using III-V material, typically separate dies for the lasers and detectors need to be bonded to have both efficient lasers and detectors [7], [8].

Recently we demonstrated a CMOS compatible approach that enables compact integration of microdisk lasers and photodetectors, by using a single III-V epitaxial structure bonded on silicon [5]. All fabrication steps for the laser and detector, except for the detector mesa, are carried out simultaneously.

In this paper, we actually demonstrate a compact optical interconnect, consisting of a directly modulated microdisk laser and a detector using this integration approach.

\section{CONCEPT}

The optical link consists of a microdisk laser and detector, both III-V based, which are connected to each other through a silicon waveguide. A microscope image of the optical link and a schematic representation of the cross-sections of the microdisk and the detector are shown in Fig. 1. The microdisk laser has a diameter of $7.5 \mu \mathrm{m}$. The detector mesa has a width of $5 \mu \mathrm{m}$ and a length of $60 \mu \mathrm{m}$. The height of the waveguide is $220 \mathrm{~nm}$ and the width is $450 \mathrm{~nm}$ such that it only supports the fundamental TE mode. For efficient coupling between the fundamental mode of the microdisk and the waveguide mode, the width of the waveguide is tapered to $750 \mathrm{~nm}$ around the disks coupling section. The same is done for the detector where the waveguide width under the detector is $500 \mathrm{~nm}$. The coupling gap between the waveguide and the laser/detector is estimated to be $\sim 250 \mathrm{~nm}$.

A single III-V epitaxial layer structure was used for both the laser and the detector. It consists of a $580 \mathrm{~nm}$ thin layer stack for the laser containing 3 compressively strained quantum wells and on top of this laser stack there is a $500 \mathrm{~nm}$ InGaAs based stack for the detector. Detailed information about this epitaxial structure can be found in [5]. As a single epitaxial structure is used, it becomes possible to make efficient lasers and detectors right next to each other and thus form an optical transceiver. This is demonstrated in this design by placing the laser and detector only $150 \mu \mathrm{m}$ apart from each other as can be seen in Fig. 1(a). As the microdisk laser in principle emits light in the clockwise and counterclockwise mode, detectors are placed at both outputs. Unidirectional operation of the microdisk lasers can be obtained by e.g. using external 

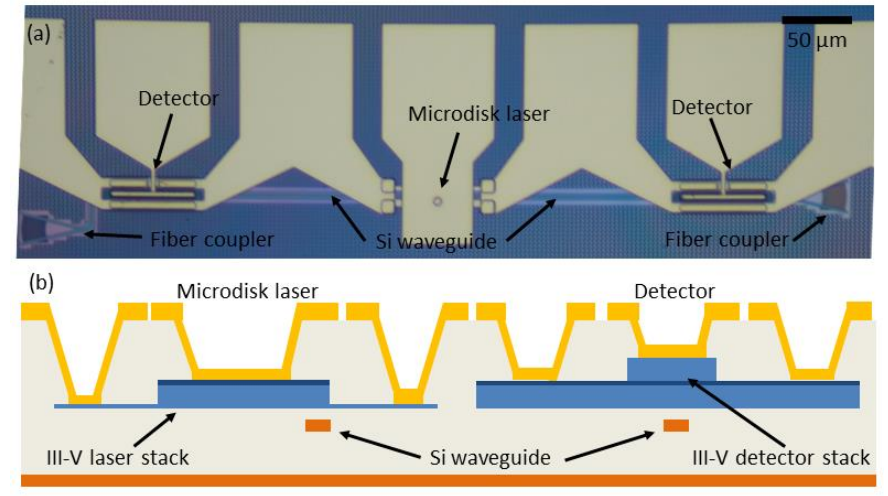

Fig. 1. Overview of the full optical link. (a) Microscope image of the link and (b) schematic representation of the cross-section of the microdisk laser and detector.

reflectors as demonstrated in [9], [10]. The waveguide on each side of the microdisk laser is terminated after the detectors with a fiber grating coupler. By doing so, it becomes possible to inject light from an external source into the detector and thus characterize the detectors separately from the laser.

\section{EXPERIMENT AND RESULT}

Before testing the full optical link, the detectors were tested by measuring the IV curve and injecting light from an external laser source into the detector via the grating couplers. The efficiency of the grating couplers was measured to be $25 \%$ at $1550 \mathrm{~nm}$. The power out of the fiber before the grating coupler was measured to be $-3.6 \mathrm{dBm}$. Taking into account the fiber coupler efficiency, the power injected into the waveguide is $109 \mu \mathrm{W}$. Fig. 2 shows the IV curves of several detectors all with the same dimensions. The performance is similar for these diodes. The dark current is around $50 \mathrm{nA}$ at a reverse bias of $1 \mathrm{~V}$ and the series resistance is around $15 \mathrm{Ohm}$. If we compare the IV curves with and without illumination we find that the responsivity of these detectors is $0.69 \mathrm{~A} / \mathrm{W}$.

The bandwidth of the detectors was measured with an Agilent N4373D Lightwave Component Analyzer (LCA). Modulated light from the LCA was injected in the detector via the grating coupler and the generated electrical signal was collected via a signal-ground (SG) high speed probe and fed back into the LCA. A bias-T was included to apply a reverse bias to the detector. The response of several detectors is shown in Fig. 3 for 0 and $-1 \mathrm{~V}$ bias. A bandwidth of $12 \mathrm{GHz}$ was obtained for the detectors when no bias was applied, while a bandwidth of $18 \mathrm{GHz}$ was measured for a bias of $-1 \mathrm{~V}$. Also, very good uniformity is obtained for the different detectors.

Next, the DC characteristics of the full optical link were measured. This was done by measuring the generated photocurrent at the detector while a current sweep was applied to the microdisk laser resulting in a current versus current characteristic. The result is shown in Fig. 4 where the black and red curves correspond with the left and right detector, respectively. The solid lines represent $\mathrm{CW}$ operation, while the dashed lines represent pulsed operation. The IV curve of the microdisk laser is also shown in this plot and from this the

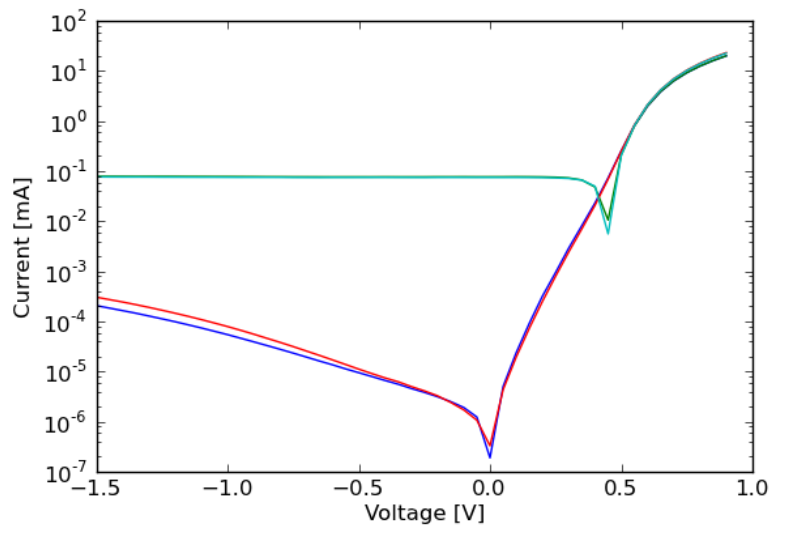

Fig. 2. IV curve of the detector with and without illumination. The responsivity is $0.69 \mathrm{~A} / \mathrm{W}$ and the dark current is $\sim 50 \mathrm{nA}$ at $-1 \mathrm{~V}$ bias.

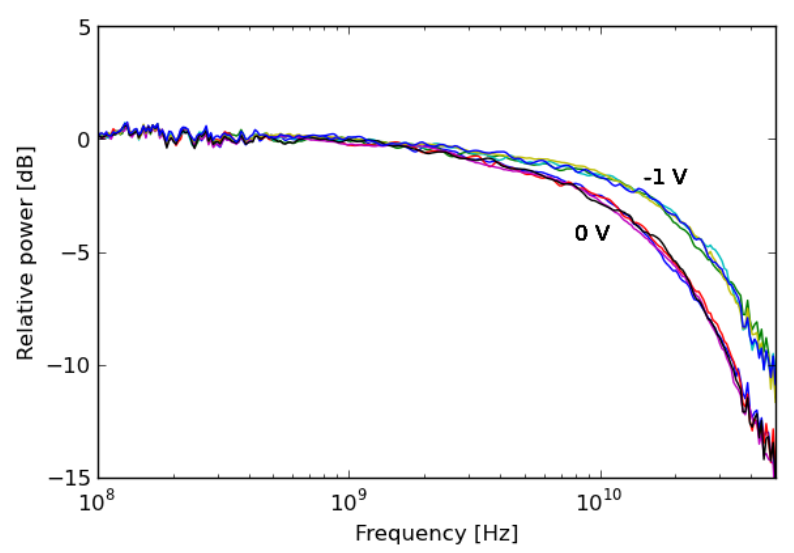

Fig. 3. Small signal response of the photodetectors for $0 \mathrm{~V}$ and $-1 \mathrm{~V}$ bias. A bandwidth of $12 \mathrm{GHz}$ and $18 \mathrm{GHz}$ was obtained for 0 and $-1 \mathrm{~V}$ bias, respectively.

series resistance of the laser is found to be around $130 \mathrm{Ohm}$. The output from the microdisk laser is bidirectional in pulsed as well as in CW mode. In CW mode, the power is evenly divided between the left and right output up to $2.3 \mathrm{~mA}$ drive current. At higher currents there is clearly some coupling between the clockwise and counterclockwise mode. The threshold current of the microdisk laser is $0.45 \mathrm{~mA}$ and the maximum generated photocurrent (single sided) is slightly above $100 \mu \mathrm{A}$. The maximum slope efficiency is $40 \mu \mathrm{A} / \mathrm{mA}$. Taking the responsivity of the detector into account this corresponds with a laser output of $145 \mu \mathrm{W}$ and a maximum slope efficiency of $57 \mu \mathrm{W} / \mathrm{mA}$. At a $2 \mathrm{~mA}$ laser drive current the generated photocurrent is 61 and $66 \mu \mathrm{A}$ for the left and right detector, respectively. The power efficiency of the optical link from the microdisk laser to one detector is therefore slightly above $3 \%$. If the microdisk laser would be made unidirectional e.g. by an external reflector as suggested above, the link efficiency could increase to $6 \%$. It is also interesting to note that the LI curve of the microdisk laser is much smoother compared to results published earlier [5] where the output of the microdisk was collected via a grating coupler. It was suggested that the oscillations in the LI curve were attributed to reflections from the grating coupler. In the present work, the 


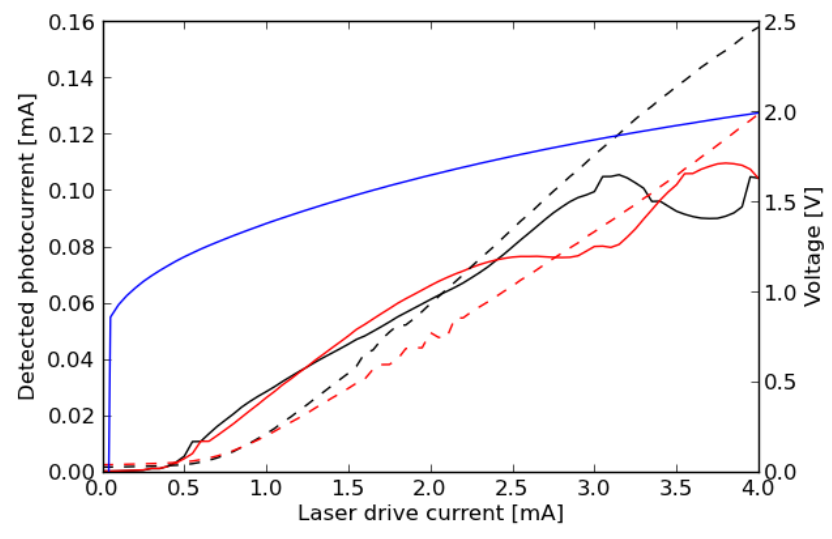

Fig. 4. Current versus current plot of the optical link. The black and red curves correspond with the left and right detector, respectively. Solid lines represent CW measurements, while dashed lines represent pulsed measurements. The IV curve is indicated by the blue curve.

facets from the detectors were designed under a 7 degree angle to avoid reflections from the III-V interface as much as possible. The smoother LI curve obtained here is another confirmation that reflections from the grating couplers, typically in the order of -15 to $-20 \mathrm{~dB}$, can disturb the performance significantly. A new grating coupler design with reflections lower than $-40 \mathrm{~dB}$ proposed by [11], would be better suited to characterize integrated lasers.

Knowing the $\mathrm{CW}$ characteristics, the small signal response of the full optical link was measured using an Agilent N5247A Performance Network Analyzer (PNA). The modulated signal from the PNA was fed into the laser via a ground-signalground (GSG) probe. Using an SG probe the detected signal at detector was fed back into the PNA. A bias-T was used for both the laser and the detector, where the detector bias was fixed to $-1 \mathrm{~V}$ and the laser bias was swept from 1.4 to $1.9 \mathrm{~V}$ (1.1 to $3.4 \mathrm{~mA}$ ) in steps of $0.1 \mathrm{~V}$. The response of the optical link versus laser bias is shown in Fig. 5 and the inset shows the $3 \mathrm{~dB}$ bandwidth versus the square root of the laser output power. As expected an almost linear relation is observed. At a bias of $1.9 \mathrm{~V} \mathrm{a} 3 \mathrm{~dB}$ bandwidth of $7.6 \mathrm{GHz}$ was measured. It should be noted that at higher frequencies the response increases again. As the distance between the laser and detector signal pads is only $120 \mu \mathrm{m}$, this is most likely caused by coupling of the microwave signal between the contacts via the sample's substrate. At this point no specific attention was paid to the impedance matching of the laser and detector to a 50 $\mathrm{Ohm}$ load. As a result, the return loss for the laser was at best $4 \mathrm{~dB}$, while for the detector it was at best $2 \mathrm{~dB}$ indicating significant reflection. Reflection problems could be avoided by using a dedicated laser driver and integrated TIA for the detector.

Finally, a large-signal modulation was applied to the optical link. Similar as for the small signal modulation, a bias-T was used where the laser was biased at $1.9 \mathrm{~V}$ while the detector was biased at -1 V. A $2^{7}-1$ PRBS pattern from an Anritsu pattern generator was used to directly modulate the laser. The modulation voltage was set to $0.4 \mathrm{Vpp}$. The generated photocurrent from the detector was fed into a $13.5 \mathrm{GHz}$

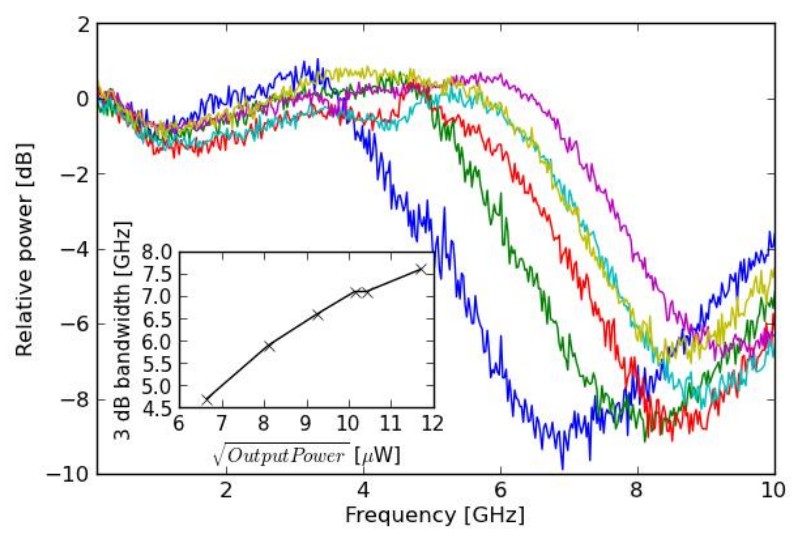

Fig. 5. Small signal response of the full optical link versus laser bias and the $3 \mathrm{~dB}$ bandwidth versus the square root of the laser output power (inset).

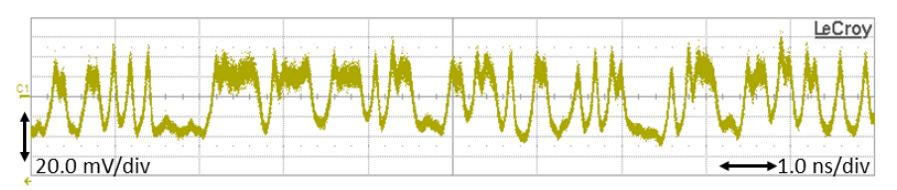

Fig. 6. Large signal modulation response of the full optical link for a $10 \mathrm{~Gb} / \mathrm{s}$ $2^{7}-1$ PRBS pattern.

electrical amplifier and collected by a $100 \mathrm{GHz}$ LeCroy scope. The large signal modulation response for a $10 \mathrm{~Gb} / \mathrm{s}$ data rate is shown in Fig. 6. A rise time with a mean value of 45 ps was measured, while the mean value of the fall time was rather high with $60 \mathrm{ps}$. Although the bit pattern can be resolved, the bit error rate is still poor at this point. It is expected that the performance can be improved by using a dedicated laser driver and integrated TIA for the detector.

\section{CONCLUSION}

We have demonstrated a low-footprint optical interconnect on SOI using heterogeneously integrated microdisk lasers and detectors. By using a single III-V epitaxial structure for both the laser and the detector spatial flexibility is ensured and a simple fabrication scheme, where both the laser and detector are processed simultaneously, can be used.

A static link efficiency slightly above $3 \%$ is achieved, which could be enhanced to $6 \%$ by making the laser unidirectional. The full optical link has a small-signal bandwidth of $7.6 \mathrm{GHz}$ and a $10 \mathrm{~Gb} / \mathrm{s}$ bit pattern could be resolved.

\section{ACKNOWLEDGMENT}

The authors would like to thank Timothy De Keulenaer and Ramses Pierco for their help in measuring the small-signal response of the full optical link.

The SOI samples were fabricated through ePIXfab.

\section{REFERENCES}

[1] D. Miller, "Device requirements for optical interconnects to silicon chips," Proceedings of the IEEE, vol. 97, no. 7, pp. 1166-1185, 2009.

[2] H. Park, A. W. Fang, R. Jones, O. Cohen, O. Raday, M. N. Sysak, M. J. Paniccia, and J. E. Bowers, "A hybrid AlGaInAs-silicon evanescent 
waveguide photodetector.," Optics express, vol. 15, no. 10, pp. 604452, May 2007.

[3] D. Feng, S. Liao, P. Dong, N.-N. Feng, H. Liang, D. Zheng, C.-C. Kung, J. Fong, R. Shafiiha, J. Cunningham, A. V. Krishnamoorthy, and M. Asghari, "High-speed Ge photodetector monolithically integrated with large cross-section silicon-on-insulator waveguide," Applied Physics Letters, vol. 95, no. 26, p. 261105, 2009.

[4] A. W. Fang, E. Lively, Y.-H. Kuo, D. Liang, and J. E. Bowers, "A distributed feedback silicon evanescent laser.," Optics express, vol. 16, no. 7, pp. 4413-9, Mar. 2008.

[5] T. Spuesens, F. Mandorlo, P. Rojo-romeo, P. Regreny, N. Olivier, J.-M. Fedeli, and D. Van Thourhout, "Compact Integration of Optical Sources and Detectors on SOI for Optical Interconnects Fabricated in a $200 \mathrm{~mm}$ CMOS Pilot Line," Journal of Lightwave Technology, vol. 30, no. 11, pp. 1764-1770, 2012.

[6] L. Chen, K. Preston, S. Manipatruni, and M. Lipson, "Integrated GHz silicon photonic interconnect with micrometer-scale modulators and detectors.," Optics express, vol. 17, no. 17, pp. 15248-56, Aug. 2009.

[7] J. Van Campenhout, P. Binetti, P. R. Romeo, P. Regreny, C. Seassal, X. J. M. Leijtens, T. De Vries, Y. S. Oei, R. P. J. Van Veldhoven, R. Notzel, L. Di Cioccio, J.-M. Fedeli, M. Smit, D. Van Thourhout, and R. Baets, "Low-footprint optical interconnect on an SOI chip through heterogeneous integration of InP-based microdisk lasers and microdetectors," Photonics Technology Letters, IEEE, vol. 21, no. 8, pp. 522-524, 2009.

[8] G. Roelkens, D. Van Thourhout, R. Baets, R. Nötzel, and M. Smit, "Laser emission and photodetection in an InP/InGaAsP layer integrated on and coupled to a Silicon-on-Insulator waveguide circuit.," Optics express, vol. 14, no. 18, pp. 8154-9, Sep. 2006.

[9] D. Liang, S. Srinivasan, D. A. Fattal, M. Fiorentino, Z. Huang, D. T. Spencer, J. E. Bowers, and R. G. Beausoleil, "Teardrop ReflectorAssisted Unidirectional Hybrid Silicon Microring Lasers," Photonics Technology Letters, IEEE, vol. 24, no. 22, pp. 1988-1990, 2012.

[10] F. Mandorlo, P. R. Romeo, X. Letartre, R. Orobtchouk, and P. Viktorovitch, "Compact modulated and tunable microdisk laser using vertical coupling and a feedback loop," Optics express, vol. 18, no. 19, pp. 19612-19625, 2010.

[11] D. Vermeulen, Y. De Koninck, Y. Li, E. Lambert, W. Bogaerts, R. Baets, and G. Roelkens, "Reflectionless grating couplers for Silicon-onInsulator photonic integrated circuits," Optics express, vol. 20, no. 20, pp. 1919-1921, 2012. 